\title{
Rethinking the origins of neurons: Q\&A with Tomasz Nowakowski
}

\author{
BY LAUREN SCHENKMAN
}

20 JANUARY 2022

Autism is thought to arise during prenatal development, when the brain is spinning its web of excitatory and inhibitory neurons, the main signal-generating cell types in the cerebral cortex. Though this wiring process remains mysterious, one thing seemed certain after two decades of studies in mice: Although both neuron types arise from radial glia, excitatory neurons crop up in the developing cortex, whereas inhibitory neurons, also known as interneurons, originate outside of the cortex and then later migrate into it.

Not so in the human brain, according to a study published in December in Nature. A team of researchers led by Tomasz Nowakowski, assistant professor of anatomy at the University of California, San Francisco, used a new viral barcoding method to trace the descendants of radial glial cells from the developing human cortex and found that these progenitor cells can give rise to both excitatory neurons and interneurons.

"This is really a paradigm-shifting finding," Nowakowski says. "It sets up a new framework for studying, understanding and interpreting experimental models of autism mutations."

Nowakowski spoke with Spectrum about the discovery's implications for studying the origins of autism in the developing brain.

\section{Spectrum: Why did you investigate this topic?}

Tomasz Nowakowski: My lab and I are interested in understanding the early neurodevelopmental events that give rise to the incredible complexity of the human cerebral cortex. We know especially little about the early stages of human development, primarily because a lot of our knowledge comes from mouse models. As we've begun to realize over the past decade, the processes that underlie development of the brain in humans and mice can be quite different. 
The key motivation comes from the findings that many psychiatric neurodevelopmental conditions, including autism, manifest very early in life and are theorized to be a consequence of disruptions occurring in these early stages of brain development.

\section{S: How did you react when your result differed so dramatically from what was previously thought?}

TN: The funny story behind this is that I didn't want to believe our results and resisted for a long time. The hypothesis that cortical radial glia generate cortical interneurons locally in primates, but not in mice, was proposed almost 20 years ago, but it has been very controversial and not generally accepted. As a scientist, I see my role as being the greatest skeptic of my own work, particularly if the findings have such long-standing implications. Therefore, the credit really goes to the trainees in my lab who pushed back and produced the evidence in support of this model.

\section{S: What implications does your discovery have for understanding autism?}

TN: What we know from studies of genetics is that loss-of-function mutations in certain genes confer a high risk for autism. What we have learned from developmental biology is that many of these genes tend to be most highly expressed during the early stages of brain development, and in the cerebral cortex in particular. One of the things that the field of autism research is trying to determine is when and where and what are the processes that are most vulnerable to mutations in those genes, with the hope of identifying these points of convergence that could be targeted therapeutically.

So if you were an autism researcher and you were interested in understanding how a particular mutation impacts the development of excitatory and inhibitory neurons in the human brain, you would typically plan to derive two different types of progenitor cells [from pluripotent stem cells] and then study how their development is affected by the mutation. Our findings suggest a new framework for studying the consequences of these mutations. A mutation could affect the generation of both excitatory and inhibitory neurons by affecting even just one population of progenitor cells.

\section{S: One theory suggests that autism results from an imbalance between excitation and inhibition in the brain. How does your discovery affect that hypothesis?}

TN: There are many ways in which such an imbalance could occur. One possibility is that the early differentiation into excitatory and inhibitory neurons is somehow affected, and the way to test that hypothesis would be to compare the generation or differentiation of these interneurons or excitatory neurons in parallel. But our findings suggest that even just a mutation disrupting normal development of cerebral cortical progenitors could affect the ratio or differentiation of these excitatory or inhibitory neurons. 
There are several studies that suggest that excitatory and inhibitory neuron generation is affected by autism mutations. When we try to explain these studies, especially those using models based on pluripotent stem cells, the assumption is that these phenotypes are a result of this patterning or organization of these stem cells into two pools: one that generates inhibitory neurons and one that generates excitatory neurons. Our finding provides an alternative interpretation.

\section{S: Tell us a bit about the viral barcoding tool you developed. Could it be used in other kinds of autism research?}

TN: We utilize viruses that are originally derived from the human immunodeficiency virus (HIV). These viruses have a high efficiency of transecting a human cell and integrating their genomic information into the DNA of individual cells, but they cannot replicate or cause any major pathology. We have engineered these viruses to deliver a molecular barcode of information, which we have predefined in the laboratory. Current technologies of high-throughput sequencing enable highly sensitive readouts of that molecular barcode information from single cells, along with the recovery of information about all the genes that these cells express. When applied to these neural stem cells, the DNA barcode integrates into the genomic DNA of a progenitor cell and is subsequently inherited by all the neurons generated by this progenitor cell. This powerful technology allows us to determine what types of neurons have been born from the same progenitor cell, and the technology can be scaled to discover thousands of these lineage relationships in a single experiment.

Our tool can be applied to essentially any experimental model, since it does not depend on transgenic mouse models or genetically modified cell lines. The barcoded virus can be applied to any experimental study to track cells over time. Moreover, it doesn't have to be restricted to tracking cell lineages. It could be also applied to other biological problems. For example, the barcoding method could be applied to other types of viruses, such as monosynaptic rabies virus, which is often used to track synaptic connections.

\section{S: Because the versatility you found doesn't occur in mice, what does that say about using mouse models to study autism?}

TN: I think that it is fair to say that if a particular autism mutation does have an impact on the local generation of inhibitory neurons in the cerebral cortex, you wouldn't be able to detect it using mice as a model. The ramification of this findings is that one would have to identify alternative models to address that question.

Embedded in this finding is an opportunity to think about what organisms other than mice could serve as in vivo experimental models.

\section{S: Is there anything else that really excites you about this discovery?}




\section{Spectrum | Autism Research News}

https://www.spectrumnews.org

TN: When we look at the mouse, at the mouse cerebral cortex, the ratio of these excitatory and inhibitory neurons is about 5 to 1 . When you look at humans and marmosets, which is another emerging animal model for human neuroscience, this ratio changes to about 2 to 1 for humans, and about 3 to 1 for marmosets. And so it really motivates you to think about what evolutionary changes between mice and humans may have caused this ratio to change so dramatically. One of the hypotheses was that perhaps the GABA-ergic interneurons could be locally generated by cortical generator cells. The evidence has gone back and forth over the years, but our study provides a strong argument that indeed this phenomenon may have been underappreciated.

\section{S: What are you going to delve into next?}

TN: I am very interested in identifying the mechanism underlying the generation of cortical interneurons by local cortical progenitor cells. The possibility that the switch between excitatory and inhibitory neuron generation could be affected by autism mutations is really exciting, and important to explore. More broadly, I am fascinated by neurodevelopmental differences between mice and humans. Identifying the experimental models that better recapitulate human neural developmental events represents a really important challenge. While there is a growing interest in using brain organoids, for example, to study these early neurodevelopmental processes, there are many important features of brain development and function that cannot be modeled in a dish.

Cite this article: https://doi.org/10.53053/FANB1055 